ISSN 1518-3483

Licenciado sob uma Licença Creative Commons

(c) (1)

\title{
Quelle évaluation pour quelle politique de formation continue en Suisse?
}

\author{
Which evaluation for which continuous vocational training \\ policy in Switzerland?
}

\author{
¿Qué tipo de evaluación para qué política de formación \\ continua en Suiza?
}

\author{
Isabel Voirol-Rubido*
}

Université de Genève, Faculté de Psychologie et des Sciences de l'éducation, Genève, Suisse

\section{Résumé}

Cet article présente une réflexion sur la politique de formation continue helvétique. II mobilise une méthodologie de recherche qualitative afin de mettre en évidence des éléments d'interprétation des décisions prises en matière de politique de formation continue ainsi que de la faible ampleur de son évaluation, en particulier à l'heure d'élaborer une toute nouvelle loi fédérale sur la formation continue en Suisse et d'intégrer

IVR : docteure en Sciences de l'éducation et économiste de l'éducation, e-mail : maria-isabel.voirol-rubido@unige.ch 
formellement ce segment au système de formation suisse suite à l'introduction d'un nouvel article sur la formation continue dans la Constitution fédérale. II ressort de cette recherche que le principe de subsidiarité des pouvoirs publics et la valorisation de la responsabilité individuelle à se former, dont est empreint le contexte suisse, contribuent à tenir à l'écart de la formation continue les pouvoirs publics, ce qui amène à une politique publique en la matière peu intrusive et une évaluation sommaire.

Mots-clés : Politique de formation continue. Évaluation. Responsabilité individuelle. Suisse.

\section{Abstract}

This article presents a reflection on the Swiss continuous vocational training policy. It mobilizes a qualitative methodology to highlight comprehensive elements of the decisions taken regarding the Swiss continuous vocational training policy as well as its low level of evaluation even though continuous vocational training has been formally integrated to the Swiss educational system and is being acted. The results show that the principle of subsidiarity which must be respected by the Swiss public authorities as well as the high status of the individual responsibility contribute to keep public authorities out of a strong continuous vocational training policy which therefore impacts the development of its evaluation.

Keywords: Continuous vocational training policy. Evaluation. Individual responsibility. Switzerland.

\section{Resumen}

Este artículo presenta una reflexión sobre la política de formación continua suiza. Moviliza una metodología de investigación cualitativa con el fin de poner en evidencia elementos de interpretación de las decisiones tomadas con respecto a la política de formación continua en Suiza así como la débil amplitud de su evaluación, particularmente a la hora de integrar formalmente el segmento de la formación continua al sistema de formación suizo mediante la introducción de un nuevo artículo en la Constitución federal y de elaborar una nueva ley federal sobre formación continua en Suiza. Los resultados de la investigación muestran el impacto del rol subsidiario atribuido al Estado suizo así como la valorización 
de la responsabilidad individual a la hora de pensar una política de formación continua en Suiza y su evaluación.

Palabras Clave: Política de formación continua. Evaluación. Responsabilidad individual. Suiza.

\section{Introduction}

A l'aulne de l'avènement de la nouvelle gestion publique (New Public Management) recherchant l'efficacité des politiques publiques (MUELLER et al., 2010) dans un contexte socioéconomique exigeant, chahuté par les crises économiques successives, assurer la qualité des systèmes de formation continue $(F C)$ prend une place centrale dès les années 90 dans les économies développées (SANTELMANN, 2007 ; TREMBLAY, 2010). Ces systèmes répondent-ils aux besoins de la société et produisent-ils les effets attendus, telles sont les questions qui s'imposent à l'heure d'évaluer leur qualité et d'affiner les politiques publiques qui la concernent. Dans le même temps émerge la prise de conscience de la nécessité de prendre en compte le caractère systémique du processus de formation (DARMON ; PEREZ, 2007, p. 47) et, par conséquent, les différents acteurs qui l'animent, pour évaluer cette qualité.

Précisons en préambule que l'évaluation de la qualité d'un système de formation peut être entreprise à différents niveaux (macro-micro), et s'intéresser à des objets aussi divers que le degré de contribution du système aux transformations du système de production, à son impact sur la progression salariale ou professionnelle des formés ou encore aux résultats en terme d'emploi (SANTELMANN, 2007) suivant le point de vue auquel on s'intéresse, qu'il s'agisse des bénéficiaires de la formation, des organismes de formation, des financeurs, des pouvoirs publics, des partenaires sociaux, des entreprises ou encore des formateurs (DARMON ; PEREZ, 2007, p. 41). 
Partant, la qualité s'appréhendera de manière différenciée, selon les critères retenus, propres à chaque partie prenante, à des moments différents du processus et suivant les points de vue de chaque acteur concerné. A ce jour, en Suisse, le processus d'évaluation du système de FC repose presque exclusivement sur une approche quantitative statistique visant à n'évaluer que l'efficacité, l'efficience et l'équité du dispositif (CSRE, 2014, p. 271-278).

D'un point de vue macroéconomique et ayant pour dessein de servir la politique publique, Gerard (2007, 2011) considère que le processus d'évaluation des systèmes de formation devrait s'intéresser non seulement à la productivité des systèmes (efficacité) et à leurs coûts (efficience), mais tenir également compte d'une dimension sociale (équité), pédagogique (spécificité des produits engendrés par les systèmes de formation et pertinence des objectifs poursuivis par le système), ainsi que conative (capacité des systèmes de formation à développer l'engagement des formés).

L'objectif de cet article est de présenter une réflexion sur la politique de FC helvétique et son évaluation en termes de résultats dans une perspective macroéconomique. Elle s'appuie sur une recherche récente portant sur la potentialité d'un cofinancement tripartite de la FC orienté vers la demande (usager de la formation) à améliorer l'accès à la FC et la faisabilité économique, politique et sociale en Suisse d'un tel modèle de financement ${ }^{1}$. Cette dernière a été réalisée pendant les discussions parlementaires visant la prochaine implantation d'une nouvelle loi fédérale sur la formation continue (LFCo) en Suisse. Ce travail a impliqué non seulement une recherche documentaire portant sur la politique de FC en Suisse et son évaluation mais également une enquête de terrain dont la richesse des données récoltées permet de mettre en évidence des éléments de compréhension de la politique de FC en Suisse, en particulier de l'orientation que les autorités fédérales suisses entendent lui donner dans le futur proche. Elle permet également de souligner l'état peu avancé

1 Les résultats de cette recherche seront publiés dans une monographie prochainement. 
du processus d'évaluation de la politique de FC en Suisse qui, malgré tout, influence la prise de décision politique.

Notre propos s'organise de la manière suivante. Après avoir brièvement explicité le dispositif de recherche ayant permis de récolter l'information sur laquelle se fonde notre réflexion sur la politique de FC en Suisse, nous présentons quelques caractéristiques du contexte socioéconomique et politique suisse permettant de cerner le contingentement dans lequel évolue la Suisse et comprendre la place réservée à la FC dans ce pays. Nous évoquons ensuite l'évolution de la législation suisse en matière de $\mathrm{FC}$ puis mettons en exergue des éléments de réflexion permettant de cerner la politique de la Suisse en matière de FC ainsi que l'ampleur restreinte de son évaluation. Le dernier point présente nos conclusions.

\section{Le dispositif méthodologique mobilisé}

Afin de comprendre la place réservée à la $\mathrm{FC}$ en Suisse et les décisions politiques prises en la matière nous avons adopté une méthodologie de recherche qualitative en nous appuyant sur une enquête de terrain sollicitant le concours d'une douzaine de partenaires sociaux concernés par la FC, à savoir les représentants des salariés, des entreprises, des pouvoirs publics et des prestataires de formation. Les informations ont été récoltées moyennant des entretiens individuels semi-directifs afin de laisser parler librement chaque interlocuteur sur la situation de la FC en Suisse et les orientations que les institutions qu'ils représentent souhaiteraient leur voir donner en recentrant, si nécessaire, l'entretien sur nos objectifs de recherche.

Nous avons ensuite procédé à une analyse des entretiens selon une approche thématique ${ }^{2}$ (BLANCHET ; GOTMAN, 2007, p. 93, 96)

2 Méthode consistant à analyser le discours par thèmes définis selon une grille d'analyse élaborée empiriquement (BLANCHET ; GOTMAN, 2007, p. 93, 97-99). 
en trois étapes. La première a consisté à faire ressortir les tendances fortes et récurrentes qui apparaissent dans les discours. Nous avons ensuite recherché les divergences de points de vue entre les acteurs pour finalement parvenir à contraster les positions des uns et des autres et ainsi laisser surgir le débat d'idées qui a lieu actuellement en Suisse, notamment dans le contexte de l'élaboration de la LFCo.

\section{Caractérisitiques politiques et socioéconomiques suisses}

La Suisse est une Confédération dotée d'un pouvoir central et constituée de 26 cantons et demi-cantons disposant chacun d'une Constitution cantonale, d'un gouvernement, d'un parlement, de tribunaux et de lois qui doivent être compatibles avec celles de la Confédération.

D'un point de vue économique, la Suisse se porte bien en comparaison internationale. Son tissu économique est en grande partie composé de PME dont plus de $90 \%$ sont des microentreprises ${ }^{3}$, c'est-àdire employant jusqu'à neuf salariés. Ce tissu économique génère l'un des taux d'activité les plus élevés des pays de l'Union européenne et de l'AELE. En effet, selon les données statistiques de l'OFS, la Suisse présente, au deuxième trimestre de 2012, un taux d'activité standardisé ${ }^{4}$ de 67,8 \% la plaçant au troisième rang de l'échantillon retenu (OFS, 2013a, p. 17) et un faible taux de chômage au sens du BIT estimé au deuxième trimestre 2012 à $3,7 \%$ (OFS, 2013a, p. 17). L'économie suisse est fortement tertiarisée (près de $70 \%$ des emplois) (OFS, 2009, p. 18) et génère une production à haute valeur ajoutée requérant une une main-d'œuvre qualifiée et de gros efforts en matière de recherche et développement ( $\& \& D)$, en particulier de la part du secteur privé. En effet, ce dernier contribue à hauteur de près

3 Information tirée du site internet de l'OFS à l'adresse : <http://www.bfs.admin.ch/bfs/portal/fr/ index/themen/06/02/blank/key/03/01.html>. Consultée le : 24 octobre 2014.

4 Le taux d'activité standardisé est le taux d'activité calculé sur la population de 15 ans et plus (OFS, 2012, p. 14). 
des trois quarts de l'effort total de R\&D (OFS, 2013b, p. 11). La bonne santé de l'économie intérieure suisse soutenue par la croissance des dépenses de consommation et du secteur de la construction participent, selon Baumberger et Gaillard (2010, p. 39-43) de la bonne santé du marché du travail suisse en comparaison internationale.

Le niveau de formation de la population suisse de 25 à 64 ans ne cesse d'augmenter (OFS, 2010, p. 38). Selon la projection de l'OFS environ $55 \%$ de la population suisse devrait atteindre un degré de formation tertiaire à l'orée 2025. A titre de comparaison, ce taux était de $35 \%$ en 2009 (OFS, 2010, p. 39). Cependant, les entreprises rencontrent des difficultés à recruter les profils de compétences correspondant à leurs besoins (OFS, 2009, p. 12) — c'était le cas de 17,1 \% des firmes en 2005 et 35,3\% d'entre elles en 2010 (OFS, 2011, p. 12).

Selon les dernières statistiques suisses portant sur la FC, près de $63 \%$ de la population résidante permanente en Suisse de 25 à 64 ans participent à la FC en 2011. En comparaison internationale, les entreprises suisses $(81 \%)$ apparaissent comme les troisièmes plus formatrices parmi celles des pays de l'Union européenne (moyenne à 66 \%) (OFS, 2013a, p. 52). Cependant, la FC profite davantage aux actifs de 35 à 44 ans $(66,5 \%)$, aux personnes hautement qualifiées $(79,4 \%)$, aux personnes professionnellement occupées $(68,2 \%)$ à des poste à responsabilité $(77,9$ \%) et à celles à temps partiel de $50 \%$ à $89 \%$ (71,8 \%), révélant ainsi des inégalités d'accès à la FC (OFS, 2013c, p. 39).

Mais au-delà des chiffres, quelle place occupe réellement la FC en Suisse?

\section{La place de la FC en Suisse}

Historiquement, l'éducation a toujours été une prérogative cantonale. Cependant, l'entrée en vigueur en 2006 de l'art. 61a de la Constitution fédérale (Cst.) impose une compétence conjointe à la Confédération et aux cantons pour l'harmonisation des législations 
cantonales en matière d'éducation. Cela se traduit notamment par un accroissement de l'engagement de la Confédération dans la FC en l'intégrant formellement au système de formation suisse (art. 64a Cst.) ce qui n'était pas le cas jusqu'alors. Forte de cette nouvelle compétence, la Confédération reçoit mandat d'élaborer une loi-cadre fédérale dédiée spécifiquement à la FC.

En Suisse, comme l'indique le rapport du Département fédéral de l'économie sur une future politique de la Confédération dans le domaine de la FC (DEPARTEMENT FEDERAL DE L'ECONOMIE, 2009, p. 3), le développement de la FC s'est fait de manière pragmatique, au gré des circonstances. Il en découle une offre de formation, tant privée que publique, très dispersée, une législation concernant la FC très diversifiée et des mesures de soutien public à son égard peu nombreuses et très diverses. Ainsi, des offres de formation d'utilité publique évoluent côte à côte avec celles à caractère lucratif qu'elles soient proposées par les entreprises et/ou les instituts de formation.

Al'heure actuelle, la FC est structurée en quatre domaines : la FCà caractère social ou de loisirs, la formation professionnelle continue (FPC), la FC du degré tertaire et la formation de rattrapage. La FC à caractère social ou de loisirs est celle qui sert des intérêts personnels mais qui peut avoir un intérêt public tels que, par exemple, la formation politique, la formation des parents, ou encore la formation en informatique, etc. La formation professionnelle continue (FPC) intervient après l'entrée dans le marché du travail et a une visée professionnelle, quelle soit de spécialisation, de réinsertion ou de reconversion. La FPC englobe la FC à des fins professionnelles organisée en fonction de la demande du marché du travail qui dépend de l'évolution des domaines d'activités. Elle est proposée par les organisations du monde du travail lorsque le contenu de la formation est spécifique et par des prestataires privés et/ou institutions publiques de formation lorsque la formation revêt un caractère général. La FPC concerne donc également la FC organisée par les entreprises, qu'elle soit interne ou externe. Cette dernière est laissée à la seule gouvernance du secteur privé. La FC du degré tertaire est le troisième segment de 
la FC. Elle est offerte par les hautes écoles (université et hautes écoles spécialisées) et est réglementée dans les lois spéciales correspondantes. Finalement, la formation de rattrapage, c'est-à-dire celle permettant d'acquérir les compétences de base manquantes, constitue le quatrième pilier de la FC en Suisse.

Jusqu'en 2006, aucune loi générale fédérale sur la FC n'existe. Elle est considérée comme un droit du travailleur et, en tant que tel, le droit du travail suisse y fait référence. Selon ce droit, la FC est de la responsabilité de l'employeur aussi longtemps qu'elle contribue à l'employabilité et à l'évolution professionnelle du salarié (GEISER, 2011, p. 7) laissant les mains libres à l'employeur de gérer l'investissement en FC comme il l'entend. En-dehors de la FC nécessaire à la pratique d'une profession (telle que, par exemple, celles liées à la santé ou à la sécurité), qui est prise en charge financièrement par l'employeur, le salarié ne dispose pour se former que d'un droit légal limité à une semaine sans obligation de l'employeur ou de l'Etat d'octroyer un financement. Précisons, que dans certaines circonstances toutefois, les conventions collectives de travail couvrent certains coûts de formation ${ }^{5}$ et, dans certains cas ${ }^{6}$ également, les coûts de formation bénéficient d'une déduction fiscale plafonnée à CHF 12'000 (aprox. BRL 32'000).

A ce jour, les dispositions fédérales concernant la FC sont intégrées dans différentes lois spéciales telles que la loi sur la formation professionnelle, la législation sur les hautes écoles, la loi sur le chômage, l'invalidité ou les étrangers. Ces règlementations portent donc sur des thématiques spécifiques et s'adressent à des publics-cibles. Par ailleurs, seuls cinq (Appenzell Rhodes-Intérieures, Fribourg, Genève, Grisons,

5 Certaines conventions collectives de travail prévoient un fonds destiné à la formation alimenté par des cotisations employeurs-employés (financement privé). Le taux de couverture des CCT a été estimé en Suisse par le syndicat USS à $50 \%$ de la population active occupée (site web <www.uss. ch/themes/travail/salaires-et-cct/article/details/la-moitie-des-salariees-protege-par-une-cct/>, consulté le $1^{\text {er }}$ octobre 2013.

6 S'agissant de formation professionnelle continue équivalente au niveau d'éducation de base (inférieure au secondaire II) certifiée. 
Valais) des 26 cantons et demi-cantons suisses disposent d'une loi ou d'une ordonnance portant exclusivement sur la FC. Les autres intègrent les dispositions sur la FC dans la législation sur la formation professionnelle. Ceci amène un déséquilibre législatif concernant la FC entre les cantons les plus favorables à la FC et les autres.

Donnant suite au projet de loi approuvé par le Parlement suisse le 15 mai 2013 et élaboré en concertation avec les cantons et les différents interlocuteurs concernés par la FC en Suisse (CONSEIL FÉDÉRAL SUISSE, 2013), une loi-cadre fédérale portant sur la FC a vu le jour le 20 juin 2014 et est sur le point d'être introduite en Suisse. Sont désormais couverts par cette loi tous les champs de la vie professionnelle, sociale, politique et culturelle sans qu'auncun contenu spécifique ne soit requis. Elle concerne autant la formation pour adultes générale que celle à des fins professionnelles pour autant qu'elle soit destinée à des adultes, quelle soit structurée en-dehors du système de formation formelle et de celui de la formation initiale, et qu'elle ne donne pas lieu à une certification reconnue par l'Etat (CONSEIL FÉDÉRAL SUISSE, 2013, p. 3308; OFFT, 2011, p. 14).

L'objectif premier de la LFCo est d'améliorer l'accès à la FC au plus grand nombre en favorisant l'initiative individuelle et en garantisant la transparence, la qualité et la perméabilité de l'espace suisse de formation (CONSEIL FÉDÉRAL SUISSE, 2013, p. 3308) tout en préservant le rôle subsidiaire de l'Etat en matière de FC (CONSEIL FÉDÉRAL SUISSE, 2013, p. 3267). C'est-à-dire que n'apparaît dans la loi aucun droit opposable à la FC pour les travailleurs ni aucune obligation légale de la part de l'Etat ou des employeurs à assumer la FC de la population active.

Cependant, prenant la mesure de l'importance des compétences de base, c'est-à-dire celles développées dans le cadre de la formation de base (lecture, écriture, mathématiques élémentaires, utilisation de la technologie de l'information et de la communication), pour le développement social et économique du pays et pour accéder à tout programme de FC, le législateur attribue la responsabilité de la formation de base au pouvoir central. Ainsi, le rôle de l'Etat fédéral se limite à faciliter l'accès à la FC aux personnes d'un niveau de formation initial inférieur 
à celui de la formation obligatoire en octroyant un financement orienté vers la demande (c'est-à-dire vers le formé). Les cantons régissent le reste de la FC avec la liberté de proposer des mesures plus favorables si elles le souhaitent. La gestion du système de $\mathrm{FC}$ reste de fait en mains cantonales bien que sa coordination intervienne au niveau fédéral.

Par ailleurs, le législateur délègue le développement et la garantie de la qualité de la formation (transparence et qualité de l'offre de formation) aux prestataires de formation tablant sur la concurrence pour garantir une offre de formation transparente et de qualité qui réponde aux besoins des demandeurs, notamment du marché de l'emploi, à moindre coûts.

La LFCo préconise également une égalité d'accès à la FC en promouvant des mesures pour garantir une égalité d'accès effective à la FC, par exemple, entre hommes et femmes, en mettant en place des mesures adaptées aux besoins des personnes diminuées, en facilitant l'intégration des étranger et l'employabilité des personnes peu qualifiées. Pour autant, sans que ne soit exigée aucune mesure concrète.

Il ressort clairement de la LFCo une volonté affichée de favoriser avant tout la responsabilité et l'initiative individuelles en matière de FC en attribuant un rôle subsidiaireàl'Etat (fédéral et cantonal) et donc en tablant sur le financement privé (employés, employeurs, conventions collectives de travail) pour soutenir la FC, restreignant ainsi l'intervention publique à la formation de base. En fin de compte, bien que l'un des objectifs de la loi ait été une plus grande implication de l'Etat fédéral en matière de FC, il ressort du texte final de la LFCo que ce dernier se cantonne à assumer le financement de la formation de base, des projets novateurs en matière de FC ainsi que de la coordination et du développement de la FC au niveau national. Pour le reste, la FC devra compter sur les ressources privées comme actuellement. En particulier concernant l'offre de formation, particulièrement éclatée et peu transparente, qui reste régulée par les lois du marché et ses travers.

Cette timidité de l'implication publique en matière de FC ancrée dans ce nouveau texte de loi nous conduit à nous intéresser aux raisons d'une portée restreinte de l'évaluation de la politique de FC en Suisse et à 
celles qui ont contribué à ce que la Suisse s'éloigne encore davantage d'un droit opposable à la FC.

\section{L'évaluation de la politique de la FC en Suisse}

La dispersion de la législation concernant la FC à l'échelle nationale et cantonale en Suisse concourt à l'absence de principes communs, d'uniformité de critères d'intervention, d'instruments d'action, ou de procédure standard en matière de FC du fait que ces lois ne sont pas nécessairement assignées au champ de la formation mais concernent, par exemple, la législation du travail, de la santé, ou encore de l'immigration. Ceci rend difficile la mise en place d'une politique de FC cohérente et efficace en Suisse. Elle joue également un rôle non négligeable sur la portée réduite de l'évaluation de la politique de FC à l'échelle fédérale ainsi que, dans de nombreux cas, à l'échelle cantonale, et ce malgré sa valeur économique, politique et sociale jugée élevée en Suisse $^{7}$ (OFS, 2013a, p. 7). En Suisse, l'évaluation de la politique de FC se restreint à sa dimension productive (efficacité et efficience) et ne tient pas ou peu compte des autres dimensions préconisées par Gerard (2011), à savoir les dimensions sociales, pédagogiques et conatives.

Cette frilosité des pouvoirs publics fédéraux à évaluer la politique de FC en Suisse résulte notamment du fait que la FC relève majoritairement de la sphère privée (env. 80 \%) (CSRE, 2006, p. 183) ce qui rend difficile l'accès à l'information la concernant. Relevons à ce propos qu'à ce jour, l'information concernant la FC n'est obtenue presque exclusivement qu'à travers les enquêtes adressées à la population. Les entreprises collaborent peu dans ce type de démarche. Ce n'est d'ailleurs qu'en 2014 que la première enquête sur la FC adressée aux entreprises

7 Le marché de la FC a été estimé à environ CHF 5,3 milliards pour 2007 (approx. BRL 14,14 milliards), soit $1 \%$ du PIB du pays ou $20 \%$ des dépenses de l'Etat consacrées à l'enseignement (MESSER ; WOLTER, 2009, p. 41). 
est publiée en Suisse (OFS, 2014). Les pouvoirs publics n'interviennent dans la FC qu'à titre subsidiaire et de ce fait ne se sentent pas légitimés à appliquer une politique publique astreignante. A ce jour, l'évaluation de la politique de FC en Suisse repose presque exclusivement sur une approche quantitative statistique qui élabore, depuis une vingtaine d'années, un nombre restreint d'indicateurs issus des ESPA (enquête sur la population active). Ces dernières consacrent, tous les quatre ans, un volet dédié à la FC. De ces enquêtes ressortent avec récurrence deux constatations qui brouillent les pistes à l'heure de réfléchir à une politique de formation.

La première relève du fait qu'à l'échelle internationale la Suisse présente un taux de participation à la FC parmi les plus élevés des pays européens ce qui freine l'urgence à mettre sur pied une politique de formation pro active qui favorise l'accès à $\mathrm{FC}$ au plus grand nombre et surtout l'intervention des pouvoirs publics dans ce domaine. La seconde porte sur la persistance des inégalités d'accès à la FC. Ces inégalités contribuent à réveiller les consciences pour envisager un moyen de contrer ce sous-investissement en matière de formation, compte tenu d'un marché du travail friant de compétences de plus en plus élevées pour répondre aux demandes d'une écononomie innovante (AGHION ; HOWITT, 2010).

Certains évènements marquent cependant un pas vers une volonté de s'intéresser davantage à la FC en Suisse. Il y a tout d'abord la volonté populaire de promulguer une loi-cadre fédérale portant spécifiquement sur la FC (LFCo) qui pousse à réfléchir sur le rôle qu'y joueraient les pouvoirs publics. Il y a ensuite la volonté de rendre comparables les enquêtes statistiques suisses avec celles de ses voisins afin de jauger la performance suisse par rapport à celle d'autres pays, au risque parfois de passer outre la question de la contextualisation des résultats pourtant cruciale à l'heure d'interpréter les résultats obtenus. Cette volonté d'aligner la méthodologie d'évaluation statistique des performances de la FC en Suisse sur celle adoptée dans l'Union européenne (enquêtes CVTS) rentre dans la logique d'un pays situé géographiquement au centre du continent européen, sans toutefois avoir adhéré à l'Union européenne, partenaire commercial incontournable pour la Suisse. 


\section{Les piliers tuteurs de la nouvelle LFCo et de la politique de FC en Suisse}

Globalement, tous nos interlocuteurs reconnaissent l'importance que revêt la FC pour la bonne santé socioéconomique de la Suisse. Tous reconnaissent également le peu d'intérêt politique qu'elle suscite. Ils pensent tous qu'actuellement, l'attention est davantage portée vers la formation professionnelle initiale qui occupe, ces derniers temps, une place de choix dans les esprits.

De manière générale, deux positionnements se dégagent. D'un côté, le patronat et les autorités politiques expriment une certaine frilosité à l'heure de concéder trop de prérogatives aux pouvoirs publics en matière de FC. De l'autre, les représentants des travailleurs et les prestataires de formation voient d'un bon oeil que les autorités politiques réglementent davantage le marché de la FC pour lisser les inégalités d'accès que l'on y observe. Le patronat défend le maintien de la FC dans la sphère privée et souhaite tenir à distance toute éventuelle viscissitude d'interventionnisme étatique en matière de FC, ce d'autant au vu de la performance statistique jugée satisfaisante de la Suisse en la matière. Les pouvoirs publics, bridés par le principe de subsidiarité de l'Etat et mis sous pression par le patronat restent à distance. De l'autre, les syndicats et les prestataires de formation qui voient d'un bon oeil l'intervention publique pour enrayer les inégalités d'accès à la FC de certains publics, en particulier les publics peu qualifiés, et réguler un marché de la FC passablement éclaté et peu lisible. Les syndicats mettent néanmoins en garde contre un interventionisme étatique exacerbé moultes fois assosié à de l'inefficience économique parce que bureaucratique et coûteux.

Les raisons de cette frilosité sont diverses. Outre le fait, déjà évoqué précédemment et mis en évidence par tous nos interlocuteurs, de la bonne performance statistique de la Suisse en matière de FC à l'échelle internationale, qui n'incite pas les pouvoirs publics à prioriser leur action sur ce segment du système de FC, mettons en exergue certaines caractéristiques socioculturelles suisses qui pèsent à n'en pas douter de tout leur poids dans la manière d'envisager la politique publique en matière de FC. 
Bien qu'en Suisse, le rôle de l'Etat ait évolué ces cinquante dernières années en s'orientant vers plus de droits sociaux, il reste empreint de libéralisme économique attaché à l'esprit d'entreprise et à l'autorégulation de la société privilégiant la responsabilité individuelle à l'interventionnisme de l'Etat (SOULET, 2010, p. 36). Ceci explique, en partie du moins, la réticence de l'Etat à intervenir dans ce qui est considéré comme relevant de la sphère privée (salariés et entreprises). Précisons d'ailleurs que la vie associative et le bénévolat jouent un rôle important dans le paysage sociopolitique helvétique dans la mesure où ils agissent en complément à l'action publique que ce soit dans le repérage des problèmes sociaux ou dans leur remédiation. En matière de FC cette implication est très marquée. Les institutions qui les représentent ont d'ailleurs joué un rôle important à l'heure d'élaborer la LFCo.

Ainsi, Bertozzi, Bonoli, et Gay-des-Combes (2005) estiment que:

[...] en comparaison internationale, l'Etat social suisse reste [...] un régime essentiellement passif et protecteur face à des risques sociaux typiques de la période des Trente glorieuses. De nouveaux besoins sociaux, touchant certains groupes de la population, semblent peu ou pas du tout pris en compte. Et pourtant, les transformations de la société, durant les dernières décennies, demandent des interventions toujours plus orientées vers l'investissement social (apud SOULET, 2010, p. 31).

Parmi les facteurs influant sur le rôle de l'Etat en Suisse, figure le principe de subsidiarité c'est-à-dire que les pouvoirs publics fédéraux ne sont habilités à intervenir dans un domaine que lorsqu'une réponse à un problème, qui n'est pas de son ressort, ne trouve de réponse ni au niveau communal ni à celui cantonal. Lorsque la légitimité d'intervention est remplie par les pouvoirs publics fédéraux, notamment en cas de défaillance $\mathrm{du}$ marché, entre alors en lice le principe du compromis privilégiant la coopération entre les partenaires sociaux dans le but de réguler collectivement les tensions, ce qui confère à l'Etat fédéral un rôle de médiateur.

Ainsi, comme le relève Soulet : 
La force culturelle de la subsidiarité a ainsi joué un rôle non négligeable dans le faible enthousiasme pour des mesures législatives centralisées, pour un développement des formes obligatoires d'assurance et pour toute initiative publique pouvant être lue comme une limitation de l'initiative individuelle, expliquant en cela un retard, à tout le moins un décalage, au regard du système des Etats sociaux environnants. Mais, en même temps, le fort développement du privé social a pesé fort lourd pour faire advenir des questions sociales sur la scène publique et maintenir, même quand ce n'était plus en syntonie avec l'air du temps, la nécessité de poursuivre l'effort de développement de mesures de protection sociales parce que progressivement perçues comme un dirimante à l'idéal de subsidiarité et à ses présupposés (SOULET, 2010, p. 36).

En Suisse, l'initiative privée est privilégiée à l'action publique en matière de FC, principe d'ailleurs clairement spécifé dans la LFCo.

Cette attribution d'un rôle subsidiaire aux pouvoirs publics fédéraux en matière de $\mathrm{FC}$ contrecarre toute vélléité de la part de ces derniers de s'impliquer davantage dans ce segment de la formation en Suisse et partant, n'occupant pas de rôle de premier ordre, d'envisager réellement une politique publique de $\mathrm{FC}$ et d'en évaluer ses effets.

En revanche, lorsque des dysfonctionnements du marché sont constatés, les pouvoirs publics ont la légitimité d'intervenir. C'est le cas en matière de FC lorsque l'on constate des inégalités d'accès à la FC ou lorsque l'on souhaite hausser le niveau de formation de la population afin d'éviter des coûts publics et sociaux. Ceci conduit le Parlement fédéral suisse à attribuer aux pouvoirs publics la mission de prendre à sa charge la régulation de la formation de rattrapage. Ce d'autant que ce segment dela FC n'intéresse pas ou très peu les investisseurs privés en raison de sa faible rentabilité économique.

\section{Conclusion}

Dans le contexte socioéconomique que connaît la Suisse actuellement, plongé dans une concurrence économique exacerbée par la 
course àl'innovation etla mondialisation del'économie, la FC apparaît comme jouant un rôle incontournable dans le développement les compétences requises par le marché de l'emploi et pour permettre aux citoyens de répondre aux exigences d'une société postindustrielle en constant développement.

Les interlocuteurs rencontrés dans le cadre de notre recherche reconnaissent à l'unanimité la nécessité de lancer le débat sur une politique de FC en Suisse afin de parvenir à structurer le marché de la FC pour qu'il réponde efficacement aux besoins de l'économie et permette à tout citoyen actif d'yjouer un rôle économique et social. Force est néanmoins de constater que dans le contexte économique actuel incertain et morose, balloté de crise économique en crise économique à un rythme soutenu, les priorités sont ailleurs ce qui laisse présager que la mise en place d'une politique de FC en Suisse ne soit reportée à plus tard malgré la volonté exprimée en 2006 par le peuple et les cantons que la FC fasse partie intégrante du paysage de la formation suisse, ce qui suppose qu'elle fasse également l'objet d'une politique que l'on espère pilotée par des évaluations qui tiennent compte des dimensions productive (efficacité, efficience), sociale (équité), pédagogique et conative dont est constitué tout dispositif de formation.

\section{Références}

AGHION, P. ; HOWITT, P. L'économie de la croissance. Paris : Ed. Economica, 2010. BAUMBERGER, D. ; GAILLARD, S. Le marché du travail en Suisse : un état des lieux. La Vie économique, n. 4, p. 39-43, 2010.

BERTOZZI, F. ; BONOLI, G. ; GAY-DES-COMBES, B. La Réforme de l'Etat social en Suisse: vieillissement, emploi, conflit travail-famille. Lausanne : Presses polytechniques et universitaires romandes, 2005.

BLANCHET, A. ; GOTMAN, A. L'enquête et ses méthodes : l'entretien. Paris : Armand Colin, 2007. 
CENTRE SUISSE DE COORDINATION POUR LA RECHERCHE EN ÉDUCATION CSRE. L'éducation en Suisse. Aarau, 2006.

CENTRE SUISSE DE COORDINATION POUR LA RECHERCHE EN ÉDUCATION CSRE. L'éducation en Suisse: rapport 2014. Aarau, 2014.

DARMON, I. ; PEREZ, C. Poser un jugement sur les pratiques de conseil, d'orientation et d'accompagnement des adultes. Actualité de la formation permanente, n. 211, p. 41-47, nov./déc. 2007

GERARD, F.-M. Diagnostic, enjeux et perspectives du concept d'efficacité en formation. Actualité de la formation permanente, n. 211, p. 13-23, nov./déc. 2007. GEISER, T. La formation continue dans le droit du travail. 2011. Papier présenté à la manifestation de l'OFFT, Minusio, le 15 mai 2011.

GERARD, J.-M. L'évaluation de la qualité des systèmes de formation. Mesure et évaluation en éducation, v. 24, n. 2-3, p. 52-77, 2011.

CONSEIL FÉDÉRAL SUISSE. Message relatif à la loi fédérale sur la formation cotninue (13.038). Berne, 15 mai 2013. Disponibles sur : <http://www.sbfi. admin.ch/botschaft-f>. Consultée le : 24 oct. 2014.

MESSER, D. ; WOLTER, S. C. Les dépenses pour la formation continue en Suisse : une estimation. La Vie économique, n. 6, p. 41-44, 2009.

MUELLER, D. C. et al. Choix publics : analyse économique des décisions publiques. Bruxelles : de Boeck, 2010.

OFFICE FÉDÉRALE DE LA FORMATION PROFESSIONNELLE ET DE LA TECHNOLOGIE - OFFT. Procédure de consultation relative à une loi fédérale sur la formation continue (LFCo) : rapport explicatif du 21 octobre 2011. Berne, 2011.

OFFICE FÉDÉRAL DE LA STATISTIQUE - OFS. Indicateurs du marché du travail 2009. Neuchâtel, 2009.

OFFICE FÉDÉRAL DE LA STATISTIQUE - OFS. Les scénarios de l'évolution de la population de la Suisse 2010-2060. Neuchâtel, 2010. 
OFFICE FÉDÉRAL DE LA STATISTIQUE - OFS. Indicateurs du marché du travail 2011. Neuchâtel, 2011.

OFFICE FÉDÉRAL DE LA STATISTIQUE - OFS. Vie active et rémunération du travail : définitions. Neuchâtel, 2012.

OFFICE FÉDÉRAL DE LA STATISTIQUE - OFS. Indicateurs du marché du travail 2013 : résultats commentés pour la période 2007-2013. In: OFFICE FÉDÉRAL DE LA STATISTIQUE - OFS. Indicateurs du marché du travail. Neuchâtel, 2013a.

OFFICE FÉDÉRAL DE LA STATISTIQUE - OFS. L'apport à l'économie suisse de la recherche et développement vu par la statistique macro-économique. Neuchâtel, 2013b.

OFFICE FÉDÉRAL DE LA STATISTIQUE - OFS. La formation tout au long de la vie en Suisse. Neuchâtel, 2013c.

OFFICE FÉDÉRAL DE LA STATISTIQUE - OFS. La formation continue en entreprise en Suisse : résultats de l'enquête 2011 auprès des entreprises sur la formation professionnelle continue (SBW). Neuchâtel, 2014.

SANTELMANN, P. Quelle efficacité de la formation professionnelle continue ? Actualité de la formation permanente, n. 211, p. 5-11, nov./déc. 2007.

SOULET, M.-H. Y a-t-il un Etat social suisse ? ou les enseignements d'un régime atypique. In: VERBA, D. (Ed.). Interventions sociales et rôle de l'État : regards croisés - Algérie, Allemagne, France, Israël, Maroc, Palestine, Suisse. Rennes : Presses EHESP, 2010. p. 29-39.

TREMBLAY, P. Evaluation de la qualité de deux dispositifs scolaires. Bruxelles : Université libre de Bruxelles, 2010.

Reçu : 01/12/2014

Received: 12/01/2014

Approuvé : 16/12/2014

Approved: 12/16/2014 
